\title{
Further results on the linearization problem in discrete time: the uncontrollable case.
}

\author{
Claudia Califano* \\ * Dipartimento di Ingegneria Informatica Automatica e Gestionale \\ Antonio Ruberti Università di Roma La Sapienza, Via Ariosto \\ 25,00185 Italy (e-mail: califano@dis.uniroma1.it).
}

\begin{abstract}
The paper deals with the linearization problem of non controllable discrete time submersive systems. Following the approach recently introduced in the literature for continuous time systems in Menini et al. (2012), necessary and sufficient conditions are given for the equivalence of a discrete time (not necessarily controllable) single input system to a linear one.
\end{abstract}

Keywords: Linear equivalence, differential geometry, exponential representation

\section{INTRODUCTION}

The present paper is motivated by recent results given in Menini et al. (2012) and concerning the equivalence/ feedback equivalence to linear systems of continuous time affine systems, relaxing the assumption of controllability.

Starting from the early works of Krener (1973), Brockett (1978), Jakubczyk et al. (1980), Hunt et al. (1983), Krener et al. (1983), Respondek (1985), under the controllability assumption, the equivalence to linear systems under change of coordinates (with or without feedback) has been widely studied in the literature, both in continuous time and discrete time, as shown by the great literature on this topic (see between the others Aranda-Bricaire et al. (1996), Califano et al. (1999), Califano et al. (2010), Califano et al. (2011), Grizzle (1993), Jakubczyk (1987), Lee et al. (1987), Marino (1986), Monaco et al. (1987), Respondek et al (2008), Respondek et al. (2008a)).

The approach proposed in Menini et al. (2012), which relaxes the controllability assumption, is here extended to the discrete time context and it is shown that with respect to the continuous time case some additional conditions are needed due to the weaker structure considered. The results are stated in the geometric framework introduced in Monaco et al. (1997) to deal with discrete time systems. It is also shown how to recover the standard conditions as given in (Califano et al. (1999)) when the controllability assumption holds true. This approach shows once more how the geometric framework proposed in Monaco et al. (1997), Monaco et al. (2007) is a valid tool for the analysis of the structural properties of discrete time systems.

The paper is organized as follows. In Section 1 some recalls on discrete time systems and their exponential representation are given. In Section 2 the conditions under which a given dynamics is equivalent under change of coordinates to a linear one are given. Section 3 concerns instead with the linear feedback equivalence problem. Again necessary and sufficient conditions are given. As an example in Section 4 sampled data systems are considered, with respect to the linear equivalence problem. Some concluding remarks end the paper.

\subsection{Recalls on discrete-time systems}

In the following we will consider single input nonlinear discrete-time dynamics of the form

$$
x_{k+1}=F\left(x_{k}, u_{k}\right)
$$

where the state $x \in \mathcal{R}^{n}$, while the input $u \in \mathcal{R}$; $\left(x_{k, 0}, u_{k, 0}\right)=(0,0)$ is an equilibrium pair for $(1)$, the function $F: \mathcal{R}^{n} \times \mathcal{R} \rightarrow \mathcal{R}^{n}$ is analytic in its arguments. Without loss of generality it will be assumed throughout the paper that the system is submersive, that is

$$
\text { A) }\left.\quad \operatorname{rank}\left(\frac{\partial F(x, u)}{\partial x}, \frac{\partial F(x, u)}{\partial u}\right)\right|_{\left(x_{k, 0}, u_{k, 0}\right)}=n \text {. }
$$

It should be noted that such an assumption, common in the discrete-time context, ensures the existence of a regular static state feedback $u=\gamma(x, v)$, such that the modified drift $\tilde{F}(x, \gamma(x, 0))$ is characterized by a full rank Jacobian, locally around the origin, which guarantees the invertibility of the drift for the modified system.

In the following $L_{f} \tau:=\frac{\partial \tau}{\partial x} f$, and $a d_{\tau_{1}} \tau_{2}:=\left[\tau_{1}, \tau_{2}\right]=$ $L_{\tau_{1}} \tau_{2}-L_{\tau_{2}} \tau_{1}$ will denote the Lie derivative of $\tau$ along $f$ and the Lie bracket of $\tau_{1}$ and $\tau_{2}$ respectively.

Let us now recall that in Monaco et al. (1997) it was shown that the existence of an analytic function $G^{0}(., u)$ satisfying the partial differential equations

$$
G^{0}(F(x, u), u)=\frac{\partial(F(x, u))}{\partial u},
$$

ensures the existence of the exponential representation for the drift $F(x, u)=x^{+}(u)$, given by

$$
\begin{aligned}
x^{+}(u) & =\left.e^{u \mathcal{G}(x, u)}(I d)\right|_{x^{+}(0)} \\
x^{+}(0) & =F_{0}(x) .
\end{aligned}
$$

Consider the development of $G^{0}(x, u)$ with respect to $u$ :

$$
G^{0}(x, u):=G_{1}^{0}(x)+\sum_{s \geq 1} \frac{u^{s}}{s !} G_{s+1}^{0}(x) .
$$


$u \mathcal{G}(x, u)$ in $(3)$ admits the series development (for more details see Monaco et al. (2004), Monaco et al. (2007))

$$
u \mathcal{G}(x, u)=\sum_{p>1} \frac{u^{p}}{p !} B_{p},
$$

where the coefficients $B_{p}$ are homogeneous Lie polynomials of degree $p$ in the $G_{j}$ 's. For the first terms one gets that

$$
B_{1}=G_{1}^{0}, \quad B_{2}=G_{2}^{0}, \quad B_{3}=G_{3}^{0}+\frac{1}{2}\left[G_{1}^{0}, G_{2}^{0}\right] \cdots
$$

If the drift is invertible $G^{0}(x, u)$ satisfying (2) is unique and is given by

$$
G^{0}(x, u):=\left.\frac{\partial(F(x, u))}{\partial u}\right|_{x=F^{-1}(x, u)}
$$

In this framework, given a vector field $\tau(x)$, its transport along the drift $F_{0}$, when it exists, is defined as the vector field $\tau^{1}(x):=A d_{F_{0}(x)} \tau(x)$ satisfying the relation

$$
\frac{\partial F_{0}}{\partial x} \tau(x)=\left.\tau^{1}(x)\right|_{F_{0}}
$$

If the drift term is invertible such a transport exists, is unique, and can be computed as

$$
\tau^{1}(x):=A d_{F_{0}} \tau(x)=\left.\left(\frac{\partial F_{0}}{\partial x} \tau(x)\right)\right|_{F_{0}^{-1}}
$$

In particular $G_{s}^{p}(\cdot)$ will denote the transport of $G_{s}^{0}(\cdot)$ along $F_{0} p$ times, that is

$$
G_{s}^{p}(\cdot):=A d_{F_{0}}\left(G_{s}^{p-1}\right)=A d_{F_{0}}^{p}\left(G_{s}^{0}(\cdot)\right)
$$

where $A d_{F_{0}}^{p}:=A d_{F_{0}} \circ \cdots \circ A d_{F_{0}} ; p$-times.

In a similar vein one can define $A d_{F(x, u)} \tau(x)$ the transport of $\tau(x)$ along $F(x, u)$. If the drift is invertible then it can be uniquely computed as

$$
\begin{aligned}
& A d_{F(x, u)} \tau(x)=\left.\left(\frac{\partial F(x, u)}{\partial x} \tau\right)\right|_{F^{-1(x, u)}}=e^{-a d_{u \mathcal{G}(x, u)}} A d_{F_{0}} \tau(x) \\
& =A d_{F_{0}} \tau-\left[u \mathcal{G}(., u), A d_{F_{0}} \tau\right]+\frac{1}{2}\left[u \mathcal{G}(., u)\left[u \mathcal{G}(., u), A d_{F_{0}} \tau\right]\right]-\cdots
\end{aligned}
$$

where $u \mathcal{G}(x, u)$ is given by (4).

Since the paper addresses the problem of the equivalence under change of coordinates, it is fundamental to understand the action of a change of coordinates on a discrete time system. The following result holds true.

Proposition 1. (Monaco et al. (1997)) Consider the discrete time dynamics

$$
x_{k+1}=F\left(x_{k}, u_{k}\right)
$$

to which is associated the difference differential representation

$$
\begin{aligned}
\frac{\partial x^{+}(u)}{\partial u} & =G^{0}\left(x^{+}(u), u\right) \\
x^{+}(0) & =F_{0}(x)
\end{aligned}
$$

Under the change of coordinates $z=\phi(x)$ let the transformed dynamics be

$$
z_{k+1}=\tilde{F}\left(z_{k}, u_{k}\right)
$$

with associated the difference differential representation

$$
\begin{aligned}
\frac{\partial z^{+}(u)}{\partial u} & =\tilde{G}^{0}\left(z^{+}(u), u\right) \\
z^{+}(0) & =\tilde{F}_{0}(z) .
\end{aligned}
$$

Then denoting by $\phi^{-1}(z)$ the inverse of $\phi(x)$,

$$
\tilde{G}^{0}(z, u)=\left.\left(\frac{\partial \phi(x)}{\partial x} G^{0}(x, u)\right)\right|_{\phi^{-1}(z)}
$$

and

$$
\tilde{F}_{0}(z)=\left.\phi\left(F_{0}(x)\right)\right|_{\phi^{-1}(z)}
$$

We end this section by recalling two results stated in Menini et al. (2012), and which play a key role in the present paper.

Theorem 1. (Cicogna et al. (1999), Menini et al. (2011)) Let $\tau(x) \in \mathbb{R}^{n}$ be analytic at $x=0, \tau(0)=0$, and $\left.\frac{\partial \tau}{\partial x}\right|_{x=0}=K$ semi simple (that is diagonalizable). If the eigenvalues of $K$ belong to the Poincaré domain (i.e. the convex hull of the $n$ points $\lambda_{1}, \cdots, \lambda_{n}$ in the complex plane does not contain the origin of $\mathbb{C}$ ) then there exists a near-identity diffeomorphism ${ }^{1} z=\phi(x)$ such that $\tilde{\tau}(z)=$ $\left.\left(\frac{\partial \phi}{\partial x} \tau(x)\right)\right|_{\phi^{-1}(z)}$ is in the Poincaré-Dulac normal form. If in addition, there are no resonances among the eigenvalues of $K$ (that is for any choice of $n$ integers $k_{i} \geq 0, i \in[1, n]$ such that $\left.\sum_{i=1}^{n} k_{i} \geq 2, \sum_{j=1}^{n} \lambda_{j} k_{j} \neq \lambda_{k} \forall k \in[1, n]\right)$, then $\tilde{\tau}(z)=K z$ that is, it is linear.

Lemma 1. (Menini et al. (2012)) Given a vector field $h(x) \in \mathbb{R}^{n}$ and two scalar functions $\eta_{i}(x) \in \mathbb{R}, i \in[1,2]$, consider the partial differential equation in the scalar unknown $a(x) \in \mathbb{R}$ :

$$
L_{h} a(x)=\eta_{2}(x) a(x)+\eta_{1}(x)
$$

If $h, \eta_{1}$ and $\eta_{2}$ are analytic at $x=0$ and $h(0) \neq 0$, then for any boundary condition $a\left(0, x_{2}, \cdots, x_{n}\right)=k\left(x_{2}, \cdots, x_{n}\right)$, $k$ analytic at $x=0$, the partial differential equation (9) admits a solution $a(x)$ analytic at $x=0$.

\section{LINEAR EQUIVALENCE}

In the present section the conditions for the equivalence of a discrete time submersive system, to a linear one under change of coordinates are given. The following result holds true.

Theorem 2. There exists a diffeomorphism $z=\phi(x)$ such that system (1) is equivalent to a linear system if and only if it admits a difference differential representation of the form (6) where

i.) $G^{0}(x, u)=G_{1}^{0}(x)$

and there exists a vector field $\tau(x)$ analytic in $x=0$ such that $\tau(0)=0,\left.\frac{\partial \tau}{\partial x}\right|_{x=0}=I d$ which satisfies the following conditions:

ii.) $A d_{F_{0}} \tau=\tau$

iii.) $\left[G_{1}^{0}, \tau\right]=G_{1}^{0}$.

Proof. Assume that the system is equivalent to a linear system under the change of coordinates $z=\phi(x)$. Without loss of generality we can assume that $\left.\frac{\partial \phi(x)}{\partial x}\right|_{x=0}=I d$ and

\footnotetext{
1 a diffeomorphism $z=\phi(x)$ is called a near-identity diffeomorphism, if $\phi(0)=0$ and its Jacobian computed in $x=0$ coincides with the identity matrix
} 
denote by $\phi^{-1}(z)$ the inverse function of $\phi(x)$. In the new coordinates

$$
z_{k+1}=A z_{k}+B u_{k}
$$

A possible difference differential representation associated to $(10)$ is

$$
\begin{aligned}
\tilde{G}^{0}(z, u) & =B \\
z^{+}(0) & =A z
\end{aligned}
$$

As a consequence, according to (7)

$$
G^{0}(x, u)=\left.\left(\frac{\partial \phi^{-1}(z)}{\partial z} B\right)\right|_{z=\phi(x)}=G_{1}^{0}(x)
$$

which proves that there exists at least one representation of the system satisfying i), that is the independence of $G^{0}(x, u)$ from $u$.

Take now in the $z$-coordinates the vector field $\tilde{\tau}(z)=$ $z$. Such a vector field satisfies $\tilde{\tau}(0)=0,\left.\frac{\partial \tilde{\tau}}{\partial z}\right|_{z=0}=$ Id. In the $x$-coordinates it is transformed into $\tau(x)=$ $\left.\left(\frac{\partial \phi^{-1}(z)}{\partial z} \tilde{\tau}(z)\right)\right|_{z=\phi(x)}$ and due to the properties of $z=$ $\phi(x), \tau(x)$ also satisfies $\tau(0)=0,\left.\frac{\partial \tau(x)}{\partial x}\right|_{x=0}=I d$.

By assumption we have that in the $z$-coordinates, $\tilde{F}_{0}(z)=$ $A z$ so that it is easily verified that

$$
A d_{\tilde{F}_{0}(z)} \tilde{\tau}(z)=\tilde{\tau}(z)=z
$$

As a consequence in the $x$-coordinates denoting by $\tau(x)$ the transformed vector field $\tau(x)=\left.\left(\frac{\partial \phi^{-1}(z)}{\partial z} \tilde{\tau}(z)\right)\right|_{z=\phi(x)}$, we get

$$
\begin{gathered}
\left.\left(A d_{F_{0}(x)} \tau(x)\right)\right|_{F_{0}(x)}= \\
=\left(\left.\left.\frac{\partial \phi^{-1}(z)}{\partial z}\right|_{\tilde{F}_{0}(z) \circ \phi(x)} \frac{\partial \tilde{F}_{0}(z)}{\partial z}\right|_{\phi(x)} \frac{\partial \phi(x)}{\partial x} \tau(x)\right) \\
=\left.\left(\left.\frac{\partial \phi^{-1}(z)}{\partial z}\right|_{\tilde{F}_{0}(z)} \frac{\partial \tilde{F}_{0}(z)}{\partial z} \tilde{\tau}(z)\right)\right|_{z=\phi(x)}=\left.\left(\frac{\partial \phi^{-1}(z)}{\partial z} \tilde{\tau}(z)\right)\right|_{\phi\left(F_{0}(x)\right)}
\end{gathered}
$$

that is ii). In fact the previous relation implies that $A d_{F_{0}(x)} \tau(x)=\tau(x)$. Finally in the $z$-coordinates, by considering again $\tilde{\tau}(z)=z$

$$
\left[\tilde{G}_{1}^{0}(z), \tilde{\tau}(z)\right]=[B, z]=B=\tilde{G}_{1}^{0}(z)
$$

As a consequence

$$
\begin{aligned}
{\left[G_{1}^{0}(x), \tau(x)\right] } & =\left.\left(\frac{\partial \phi^{-1}(z)}{\partial z}\left[\tilde{G}_{1}^{0}(z), \tilde{\tau}(z)\right]\right)\right|_{\phi(x)} \\
& =\left.\left(\frac{\partial \phi^{-1}(z)}{\partial z} \tilde{G}_{1}^{0}(z)\right)\right|_{\phi(x)}=G_{1}^{0}(x)
\end{aligned}
$$

which proves the necessity of iii).

Sufficiency. Assume that there exists a vector field $\tau(x)$ such that $\tau(0)=0$ and $\left.\frac{\partial \tau(x)}{\partial x}\right|_{x=0}=I d$ and conditions ii) and iii) are satisfied. Then, according to Theorem 1, since $\tau(0)=0$ and $\left.\frac{\partial \tau(x)}{\partial x}\right|_{x=0}=I d$ there exists $z=\phi(x)$ such that in the new coordinates $\tilde{\tau}(z)=z$. In these new coordinates we get that

$$
\begin{aligned}
\frac{\partial \tilde{F}_{0}(z)}{\partial z} z & =\left.\left.\frac{\partial \phi}{\partial x}\right|_{F_{0} \circ \phi^{-1}(z)} \frac{\partial F_{0}}{\partial x}\right|_{\phi^{-1}(z)} \frac{\partial \phi^{-1}(z)}{\partial z} z \\
& =\left.\left.\frac{\partial \phi}{\partial x}\right|_{F_{0} \circ \phi^{-1}(z)}\left(\frac{\partial F_{0}}{\partial x} \tau(x)\right)\right|_{\phi^{-1}(z)} \\
& =\left.\left(\frac{\partial \phi}{\partial x} \tau(x)\right)\right|_{F_{0} \circ \phi^{-1}(z)}=\left.\tilde{\tau}(z)\right|_{\tilde{F}_{0}(z)}=\tilde{F}_{0}(z) .
\end{aligned}
$$

The relation $\frac{\partial \tilde{F}_{0}(z)}{\partial z} z=\tilde{F}_{0}(z)$ immediately proves that $\tilde{F}_{0}(z)=A z$.

Similarly, it is a matter of computation to verify that

$$
\begin{aligned}
{\left[\tilde{G}_{1}^{0}(z), \tilde{\tau}(z)\right] } & =\left.\left(\frac{\partial \phi}{\partial x}\left[G_{1}^{0}(x), \tau(x)\right]\right)\right|_{\phi^{-1}(z)} \\
& =\left.\left(\frac{\partial \phi}{\partial x} G_{1}^{0}(x)\right)\right|_{\phi^{-1}(z)}=\tilde{G}_{1}^{0}(z)
\end{aligned}
$$

so that one gets that

$$
\left[\tilde{G}_{1}^{0}(z), \tilde{\tau}(z)\right]=\left[\tilde{G}_{1}^{0}(z), z\right]=\tilde{G}_{1}^{0}(z)-\frac{\partial \tilde{G}_{1}^{0}(z)}{\partial z} z=\tilde{G}_{1}^{0}(z)
$$

that is $\frac{\partial \tilde{G}_{1}^{0}(z)}{\partial z} z=0$, which proves that $\tilde{G}_{1}^{0}(z)$ is constant and equal to $B$. Finally the first condition implies that $\frac{\partial \tilde{F}(z, u)}{\partial u}=B$ which ends the proof. $\triangleleft$

Remark. In the continuous time case the corresponding condition of i) is implicitely satisfied by considering dynamics affine in the control. $\triangleleft$

Based on this remark, the equivalence to a weaker structure can be obtained if the following conditions are satisfied

Theorem 3. There exists a diffeomorphism $z=\phi(x)$ such that in the new coordinates system (1) reads

$$
z_{k+1}=A z_{k}+B\left(u_{k}\right)
$$

if and only if it admits a difference differential representation of the form (6) for which there exists a vector field $\tau(x)$ such that $\tau(0)=0,\left.\frac{\partial \tau}{\partial x}\right|_{x=0}=I d$ satisfying

i. $A d_{F_{0}} \tau=\tau$

ii. $\left[G^{0}(x, u), \tau\right]=G^{0}(x, u), \forall u \in \mathcal{U}_{0}$.

Proof. We only have to prove the sufficiency of ii.). In fact in the $z$-coordinates in which $\tilde{\tau}(z)=z$ we get that, according to ii.)

$$
\left[\tilde{G}^{0}(z, u), z\right]=\tilde{G}^{0}(z, u)-\frac{\partial \tilde{G}^{0}(z, u)}{\partial z} z=\tilde{G}^{0}(z, u)
$$

which immediately implies that $\frac{\partial \tilde{G}^{0}(z, u)}{\partial z} z=0$, and consequently that $\frac{\partial \tilde{G}^{0}(z, u)}{\partial z}=B(u) . \triangleleft$

Let us end this section by noting that in the controllable case using the geometric framework of (Monaco et al. (1997)), used in Califano et al. (1999) to address the feedback linearization problem, the necessary and sufficient conditions for the equivalence to a linear system can be stated as follows:

Theorem 4. The submersive system (1) is equivalent to a controllable linear system if and only if the system admits a difference differential representation of the form (6) with 
1) $G^{0}(x, u)=G_{1}^{0}(x)$

and such that for $k \in[1, n]$, there exist $G_{1}^{k}(x)=$ $A d_{F_{0}}^{k} G_{1}^{0}(x)$ satisfying

2) $\left[G_{1}^{0}(x), G_{1}^{k}(x)\right]=0, \forall k \in[1, n]$

$3) \operatorname{rank}\left(G_{1}^{0}, \cdots, G_{1}^{n-1}\right)=n$

The desired change of coordinates $z=\phi(x)$ satisfies $\left(\frac{\partial \phi(x)}{\partial x}\right)^{-1}=\left(G_{1}^{0}, \cdots, G_{1}^{n-1}\right)$.

It can be easily verified that for controllable systems Theorem 2 implies Theorem 4 by noting that since the system is linear

$$
A d_{F_{0}}^{k}\left[G_{1}^{0}, \tau\right]=\left[G_{1}^{k}, A d_{F_{0}}^{k} \tau\right]=\left[G_{1}^{k}, \tau\right]=G_{1}^{k}, \quad \forall k \geq 0
$$

which shows that in the $z$-coordinates in which $\tilde{\tau}(z)=z$, $G_{1}^{k}$ is constant for all $k \geq 0$. Consequently

$$
\left[G_{1}^{0}, G_{1}^{k}\right]=0, \quad k \in[1, n]
$$

Remark. While Theorem 4 can be considered a constructive theorem, since the change of coordinates can be recovered by using the fact that $\left(\frac{\partial \phi(x)}{\partial x}\right)^{-1}=\left(G_{1}^{0}, \cdots, G_{1}^{n-1}\right)$, Theorem 2 clarifies some properties of the change of coordinates, since the vector field $\tau(x)$ is exactly the searched coordinates transformation. As a matter of fact, for controllable systems, which are equivalent to linear systems $\tau(x)$ satisfies

$$
\frac{\partial \tau(x)}{\partial x}=\left[G_{1}^{0}(x), \cdots, G_{1}^{n-1}(x)\right]_{x=0}\left[G_{1}^{0}(x), \cdots, G_{1}^{n-1}(x)\right]^{-1} .
$$

\section{FEEDBACK LINEARIZATION}

In the present section the problem of the equivalence under change of coordinates and regular static state feedback is addressed. The following result holds true.

Theorem 5. Consider system (1) and assume without loss of generality that the drift term $F_{0}$ is locally invertible. Then there exists a diffeomorphism $z=\phi(x)$ and a regular analytic static state feedback $u=\gamma(x, v)$ such that the given system is feedback equivalent to a linear system if and only if there exists a function $\Gamma(x, u)$ such that

$$
\text { i.) } G^{0}(x, u)=G_{1}^{0}(x) \Gamma(x, u)
$$

and there exists a vector field $\tau_{\epsilon}(x)=\tau(x)+\epsilon \tau_{1}(x)$ analytic in $x=0$ such that $\tau(0)=0,\left.\frac{\partial \tau}{\partial x}\right|_{x=0}=I d, \tau_{1}(0) \neq 0$, $\left[\tau_{1}(x), \tau(x)\right]=\tau_{1}(x)$, and satisfying for all $\epsilon \in \mathbb{R}$

ii.) $A d_{F_{0}} \tau_{\epsilon}=\tau_{\epsilon}(x)+G_{1}^{0}(x) \theta(x, \epsilon)$

iii.) $\left[G_{1}^{0}, \tau_{\epsilon}(x)\right]=G_{1}^{0}(x) \psi(x, \epsilon)$

for some $\theta(x, \epsilon)$ and $\psi(x, \epsilon)$ analytic at $x=0$.

Proof. Assume that there exists an analytic regular static state feedback $u=\gamma(x, v)$ such that the transformed system is equivalent to a linear system, and denote by $\gamma^{-1}(x, u)$ its inverse function. Between all the possible feedback functions which linearize the system, we will consider only those which ensure that at least one eigenvalue is in $\lambda=1$. Since the closed loop dynamics is equivalent to a linear dynamics it admits the exponential form representation (3). Denoting by $\tilde{x}^{+}(v)=\tilde{F}(x, \gamma(x, v))$, we thus have that $\tilde{G}^{0}(x, v)$ exists and Theorem 2 is satisfied. As a consequence from condition i) of Theorem $2, \tilde{G}^{0}(x, v)=\tilde{G}_{1}^{0}(x)$. Since

$$
\frac{\partial F(x, u)}{\partial u}=G^{0}(F(x, u), u)=\tilde{G}_{1}^{0}(F(x, u)) \frac{\partial \gamma^{-1}(x, u)}{\partial u}
$$

we get that

$$
G^{0}(x, u)=\tilde{G}_{1}^{0}(x) \tilde{\Gamma}(x, u)
$$

with $\tilde{\Gamma}(x, u)=\left.\frac{\partial \gamma^{-1}(x, u)}{\partial u}\right|_{F^{-1}(x, u)}$. Equation (12) computed for $u=0$ leads to $G_{1}^{0}(x)=\tilde{G}_{1}^{0}(x) \tilde{\Gamma}(x, 0)=$ $\tilde{G}_{1}^{0}(x) \beta(x)$, that is, due to the invertibility of the feedback law, $\tilde{G}_{1}^{0}(x)=G_{1}^{0}(x) \beta^{-1}(x)$, which proves i.) by setting $\Gamma(x, u)=\beta^{-1}(x) \tilde{\Gamma}(x, u)$.

From Theorem 2 consider $\tilde{\tau}(z)=z$, and let $\tau(x)$ be its transformed in the $x$-coordinates. By construction $\tau(0)=0,\left.\frac{\partial \tau}{\partial x}\right|_{x=0}=I d$. The vector field $\tau(x)$ then satisfies $\left[\tilde{G}_{1}^{0}, \tau\right]=\tilde{G}_{1}^{0}$. Furthermore since the closed-loop system has an eigenvalue in 1 then, in the coordinates $z=\phi(x)$ such that $\left.\frac{\partial \phi(x)}{\partial x}\right|_{x=0}=I d$ the corresponding eigenvector $e_{1}$ satisfies $(\tilde{A}-I) e_{1}=0$. Let $\tau_{1}(x)$ be $e_{1}$ in the $x$ coordinates, that is $\tau_{1}(x)=\left.\left(\frac{\partial \phi^{-1}(z)}{\partial z} e_{1}\right)\right|_{\phi(x)}$ and consider $\tau_{\epsilon}(x)=\tau(x)+\epsilon \tau_{1}(x)$, then, by construction

$$
\left[\tau_{1}(x), \tau(x)\right]=\left.\left(\frac{\partial \phi^{-1}(z)}{\partial z}\left[\tilde{\tau}_{1}(z), \tilde{\tau}(z)\right]\right)\right|_{\phi(x)}=\tau_{1}(x)
$$

and

$\tilde{G}_{1}^{0}=\left[\tilde{G}_{1}^{0}, \tau_{\epsilon}\right]=\left[G_{1}^{0} \beta^{-1}, \tau_{\epsilon}(x)\right]=\left[G_{1}^{0}, \tau_{\epsilon}(x)\right] \beta^{-1}-G_{1}^{0} L_{\tau_{\epsilon}} \beta^{-1}$ As a consequence

$$
\left[G_{1}^{0}, \tau_{\epsilon}(x)\right] \beta^{-1}=G_{1}^{0}\left(L_{\tau_{\epsilon}} \beta^{-1}\right)+G_{1}^{0} \beta^{-1}
$$

that is $\left[G_{1}^{0}, \tau_{\epsilon}(x)\right]=G_{1}^{0}\left(\beta L_{\tau_{\epsilon}} \beta^{-1}+1\right)=G_{1}^{0} \psi(x, \epsilon)$ which proves iii).

Finally, since $F_{0}=\tilde{F}\left(x, \gamma^{-1}(x, 0)\right)$, we have, after standard computations, that

$$
\begin{aligned}
\left.A d_{F_{0}} \tau_{\epsilon}\right|_{F_{0}}= & \left(\frac{\partial \tilde{F}\left(x, \gamma^{-1}(x, 0)\right)}{\partial x} \tau_{\epsilon}\right)=\left.\tilde{G}_{1}^{0}\right|_{F_{0}} \frac{\partial \gamma^{-1}(x, 0)}{\partial x} \tau_{\epsilon}+ \\
& +\left.\left(\left.\left(e^{-a d_{\tilde{G}_{1}^{0}(x) v}} A d_{\tilde{F}_{0}}\left(\tau_{\epsilon}\right)\right)\right|_{x=F_{0}}\right)\right|_{v=\gamma^{-1}(x, 0)}
\end{aligned}
$$

Since by Theorem 2, $A d_{\tilde{F}_{0}}\left(\tau_{\epsilon}\right)=\tau_{\epsilon}$ and $\left[\tilde{G}_{1}^{0}(x), \tau_{\epsilon}\right]=$ $\tilde{G}_{1}^{0}(x)$, then through standard computations we get that

$$
\left.A d_{F_{0}} \tau_{\epsilon}\right|_{F_{0}}=\left.\tau_{\epsilon}\right|_{F_{0}}+\tilde{G}_{1}^{0}\left(F_{0}\right)\left(\frac{\partial \gamma^{-1}(\cdot, 0)}{\partial x} \tau_{\epsilon}-\gamma^{-1}(\cdot, 0)\right)
$$

that is $A d_{F_{0}} \tau_{\epsilon}=\tau_{\epsilon}+\left.G_{1}^{0} \beta^{-1}\left(\frac{\partial \gamma^{-1}(\cdot, 0)}{\partial x} \tau_{\epsilon}-\gamma^{-1}(\cdot, 0)\right)\right|_{F_{0}^{-1}}$ and ii) follows with

$$
\theta(x, \epsilon)=\beta^{-1}(x)\left(\left.\left(\frac{\partial \gamma^{-1}(x, 0)}{\partial x} \tau_{\epsilon}\right)\right|_{F_{0}^{-1}}-\gamma^{-1}\left(F_{0}^{-1}, 0\right)\right)
$$

Sufficiency. The proof is constructive. In the coordinates $z$ in which $\tilde{\tau}(z)=z, \tilde{\tau}_{1}(z)$ is a constant vector due to the fact that $\left[\tau_{1}(x), \tau(x)\right]=\tau_{1}(x)$. Starting from the matrix 
$\psi(x, \epsilon)$ satisfying iii) let us consider the partial differential equation, in the unknown $\bar{\beta}(x)$,

$$
L_{\tau_{\epsilon}} \bar{\beta}=(\psi(x, \epsilon)-1) \bar{\beta} .
$$

By assumption, fixing an $\epsilon \neq 0$, the solution $\bar{\beta}(x)$ exists according to Lemma 1 . As a consequence we can compute the regular static state feedback $v=\bar{\gamma}(x, u)$

$$
v=\bar{\gamma}(x, u)=\int_{u} \bar{\beta}^{-1}\left(x^{+}(u)\right) \Gamma\left(x^{+}(u), u\right) d u
$$

with zero initial condition. Denote by $\hat{\gamma}(x, v)$ the inverse function of $\bar{\gamma}(x, u)$, that is such that $\hat{\gamma}(x, \bar{\gamma}(x, u))=u$, and consider the regular static state feedback $u=\hat{\gamma}(x, v)$. Setting $\bar{F}(x, v)=F(x, \hat{\gamma}(x, v))$, by construction $\bar{F}_{0}(x)=$ $F_{0}(x)$, so that the drift is locally invertible around $v=0$. After standard computations one gets that

$$
\bar{G}^{0}(\bar{F}, v)=G_{1}^{0}(\bar{F}(x, v)) \bar{\beta}(\bar{F}(x, v))
$$

that is $\bar{G}^{0}(x, v)=\bar{G}_{1}^{0}(x)=G_{1}^{0}(x) \bar{\beta}(x)$. Since $\bar{\beta}(x)$ satisfies (13), due to iii), one gets that $\forall \epsilon \in \mathbb{R}$

$$
\begin{aligned}
{\left[\bar{G}_{1}^{0}, \tau_{\epsilon}\right] } & =\left[G_{1}^{0}(x) \bar{\beta}(x), \tau_{\epsilon}\right]=\left[G_{1}^{0}(x), \tau_{\epsilon}\right] \bar{\beta}(x)-G_{1}^{0}(x) L_{\tau_{\epsilon}} \bar{\beta}(x) \\
& =G_{1}^{0}(x) \bar{\beta}(x)=\bar{G}_{1}^{0}(x)
\end{aligned}
$$

that is $\left[\bar{G}_{1}^{0}(x), \tau(x)\right]=\bar{G}_{1}^{0}(x)$, whereas $\left[\bar{G}_{1}^{0}(x), \tau_{1}(x)\right]=0$. Thus in the coordinates in which $\tilde{\tau}(z)=z$, the transformed $\hat{G}_{1}^{0}(z)=B$.

Consider now $\theta(x, \epsilon)$ satisfying ii) and $\bar{\beta}$ computed above. From Lemma 1 , there exists locally $\gamma_{0}(x)$, solution to the partial differential equation

$$
\left(\frac{\partial \gamma_{0}(x)}{\partial x} \tau_{\epsilon}\right)-\gamma_{0}(x)=\bar{\beta}^{-1}\left(F_{0}\right) \theta\left(F_{0}, \epsilon\right) .
$$

Consider the regular static state feedback $v=w+\hat{\gamma}_{0}(x)$, and denote by $\tilde{F}(x, w)=\bar{F}\left(x, w+\hat{\gamma}_{0}(x)\right)$ and accordingly $\tilde{F}_{0}(x)=\bar{F}\left(x, \hat{\gamma}_{0}(x)\right)$. We have that

$$
\begin{gathered}
\left.A d_{\tilde{F}_{0}}\left(\tau_{\epsilon}\right)\right|_{\tilde{F}_{0}}=\left.\left(\frac{\partial \bar{F}(x, v)}{\partial x} \tau_{\epsilon}+\frac{\partial \bar{F}(x, v)}{\partial v} \frac{\partial \hat{\gamma}_{0}(x)}{\partial x} \tau_{\epsilon}\right)\right|_{v=\hat{\gamma}_{0}(x)} \\
=\left.\left(\left.\left(A d_{\bar{F}(x, v)} \tau_{\epsilon}\right)\right|_{\bar{F}(x, v)}+\bar{G}_{1}^{0}(\bar{F}(x, v)) \frac{\partial \hat{\gamma}_{0}(x)}{\partial x} \tau_{\epsilon}\right)\right|_{v=\hat{\gamma}_{0}(x)}
\end{gathered}
$$

From Monaco et al. (1997)

$$
\begin{aligned}
A d_{\bar{F}(x, v)} \tau_{\epsilon}= & e^{-a d_{\bar{G}_{1}^{0} v}}\left(A d_{F_{0}} \tau_{\epsilon}\right)=A d_{F_{0}} \tau_{\epsilon}-\left[\bar{G}_{1}^{0}, A d_{F_{0}} \tau_{\epsilon}\right] v \\
& +\frac{v^{2}}{2}\left[\bar{G}_{1}^{0}\left[\bar{G}_{1}^{0}, A d_{F_{0}} \tau_{\epsilon}\right]\right]+\cdots
\end{aligned}
$$

By ii) $A d_{F_{0}} \tau_{\epsilon}=\tau_{\epsilon}+G_{1}^{0}(x) \theta(x, \epsilon)=\tau_{\epsilon}+\bar{G}_{1}^{0}(x) \bar{\beta}^{-1}(x) \theta(x, \epsilon)$. Substituting it in (17), and recalling that $\left[\bar{G}_{1}^{0}, \tau_{\epsilon}\right]=\bar{G}_{1}^{0}$, we get

$$
A d_{\bar{F}(\cdot, v)} \tau_{\epsilon}=\tau_{\epsilon}-\bar{G}_{1}^{0} v+\bar{G}_{1}^{0} e^{-\bar{G}_{1}^{0} v}\left(\bar{\beta}^{-1} \theta(\cdot, \epsilon)\right)
$$

As a consequence by noting that

$$
\begin{aligned}
e^{-\bar{G}_{1}^{0} v} & \left.\left(\bar{\beta}^{-1} \theta(\cdot, \epsilon)\right)\right|_{\bar{F}(\cdot, v)}=\left.e^{-\bar{G}_{1}^{0} v}\left(\bar{\beta}^{-1} \theta(\cdot, \epsilon)\right)\right|_{\bar{F}(\cdot, v) \circ \bar{F}_{0}^{-1} \circ \bar{F}_{0}} \\
& =\left.e^{\bar{G}_{1}^{0} v} \circ e^{-\bar{G}_{1}^{0} v}\left(\bar{\beta}^{-1} \theta(\cdot, \epsilon)\right)\right|_{\bar{F}_{0}} \\
& =\left.\left(\beta^{-1}(x) \theta(x, \epsilon)\right)\right|_{\bar{F}_{0}}=\left.\left(\bar{\beta}^{-1} \theta(\cdot, \epsilon)\right)\right|_{F_{0}}
\end{aligned}
$$

we get, by substituting (18) in (16), that

$$
\begin{gathered}
\left.A d_{\tilde{F}_{0}}\left(\tau_{\epsilon}\right)\right|_{\tilde{F}_{0}}= \\
\tau_{\epsilon}\left(\tilde{F}_{0}\right)+\bar{G}_{1}^{0}\left(\tilde{F}_{0}\right)\left(-\hat{\gamma}_{0}+\bar{\beta}^{-1}\left(F_{0}\right) \theta\left(F_{0}, \epsilon\right)+\frac{\partial \hat{\gamma}_{0}(x)}{\partial x} \tau_{\epsilon}\right) .
\end{gathered}
$$

Since $\hat{\gamma}_{0}$ has been computed in order to satisfy equation (15), it immediately follows that $A d_{\tilde{F}_{0}}\left(\tau_{\epsilon}\right)=\tau_{\epsilon}$, which, being satisfied for all $\epsilon$ implies that

$$
\begin{aligned}
A d_{\tilde{F}_{0}} \tau(x) & =\tau(x) \\
A d_{\tilde{F}_{0}} \tau_{1}(x) & =\tau_{1}(x)
\end{aligned}
$$

Equation (19) implies that in the $z$-coordinates in which $\tilde{\tau}(z)=z, \tilde{F}_{0}(z)=A z$, while $(20)$ implies that $\tilde{\tau}_{1}(z)=e_{1}$ the eigenvector associated to the eigenvalue $\lambda=1$.

Finally under the new feedback $v=\hat{\gamma}(x, w)=\hat{\gamma}_{0}(x)+w$ we get that

$$
\begin{aligned}
\tilde{G}^{0}(\tilde{F}(x, w), w) & =\frac{\partial \tilde{F}(x, w)}{\partial w}=\left.\frac{\partial \bar{F}(x, v)}{\partial v}\right|_{v=\hat{\gamma}(x, w)} \frac{\partial \hat{\gamma}(x, w)}{\partial w} \\
& =\bar{G}_{1}^{0}(\tilde{F}(x, w))
\end{aligned}
$$

We can thus set $\tilde{G}^{0}(x, w)=\bar{G}_{1}^{0}(x)$ and the result is proved. $\triangleleft$

\section{AN EXAMPLE: LINEAR EQUIVALENCE OF SAMPLED DATA SYSTEMS}

As an example we recover the conditions for the equivalence of a sampled data single input system to a linear one under change of coordinates, which generalize the well known results obtained in Arapostathis et al. (1989) under the controllability assumption which is here relaxed. Let us preliminary recall (Monaco et al. (1997), Monaco et al. (2007)) that given the continuous time system

$$
\dot{x}=f(x)+g(x) u
$$

the equivalent discrete time model obtained by sampling with constant rate $\delta$, is given by

$$
\begin{aligned}
& x[(k+1) \delta]=\left.e^{\delta(f+g u)}(I d)\right|_{x(k \delta)} \\
& =\left.\left(I d+\delta(f+g u)+\frac{\delta^{2}}{2} L_{f+g u}(f+g u)+\cdots\right)\right|_{x(k \delta)}
\end{aligned}
$$

The following result can the be immediately stated:

Theorem 6. There exists a diffeomorphism $z=\phi(x)$ such that the sampled system

$$
x[(k+1) \delta]=\left.e^{\delta(f+g u)}(I d)\right|_{x(k \delta)}
$$

is equivalent to a linear system if and only if the continuous time system is linear, that is there exists $\tau(x)$ such that $\tau(0)=0,\left.\frac{\partial \tau}{\partial x}\right|_{x=0}=I d$ satisfying $[f, \tau]=0$ and $[g, \tau]=g$.

Proof. We have to show the only if part, since the sufficiency is obvious. Assume that the sampled system is equivalent under sampling to a linear system. Then according to Theorem 2 there exists a $\tau(x, \delta)$ such that

$$
A d_{F_{0}} \tau=e^{-a d_{\delta f}}(\tau)=\tau-\delta a d_{f} \tau+\frac{\delta^{2}}{2} a d_{f}^{2} \tau+\ldots=\tau
$$


By considering the development of $\tau(x, \delta)$ with respect to $\delta$, that is $\tau=\tau_{0}+\delta \tau_{1}+\frac{\delta^{2}}{2} \tau_{2}+\cdots$. the previous relation leads to

$$
-\delta a d_{f}\left(\tau_{0}+\delta \tau_{1}+\cdots\right)+\frac{\delta^{2}}{2} a d_{f}^{2}\left(\tau_{0}+\delta \tau_{1}+\cdots\right)+\cdots=0
$$

since such a relation must be satisfied for any $\delta$ in a neighborhood of 0 we get $\left[f, \tau_{k}\right]=0 \forall k \geq 0$, which also implies $[f, \tau]=0$. Consider now $\left[G_{1}^{0 \delta}, \tau\right]=G_{1}^{0 \delta}$.

Again substituting the expression of $G_{1}^{0 \delta}$ we get

$\left[g-\frac{\delta}{2} a d_{f} g+\frac{\delta^{2}}{3 !} a d_{f}^{2} g-\cdots, \tau\right]=g-\frac{\delta}{2} a d_{f} g+\frac{\delta^{2}}{3 !} a d_{f}^{2} g-\cdots$ that is

$[g, \tau]-\frac{\delta}{2} a d_{f}[g, \tau]+\frac{\delta^{2}}{3 !} a d_{f}^{2}[g, \tau]-\cdots=g-\frac{\delta}{2} a d_{f} g+\frac{\delta^{2}}{3 !} a d_{f}^{2} g-\cdots$

Again iteratively, by considering the development of $\tau(x, \delta)$ with respect to $\delta$, this leads to

$$
\left[g, \tau_{0}\right]=g, \quad\left[g, \tau_{k}\right]=0 \quad \forall k \geq 1
$$

As a consequence the original system is linear since $\left.\frac{\partial \tau(x, \delta)}{\partial x}\right|_{x=0}=I d$ for all $\delta$ in a neighborhood of 0 . This in particular it implies that $\left.\frac{\partial \tau_{0}(x)}{\partial x}\right|_{x=0}=I d . \triangleleft$

\section{SOME CONCLUDING REMARKS}

In the present paper, the problem of the equivalence of a nonlinear discrete time submersive single input system to a linear one, under change of coordinates, or regular static state feedback and change of coordinates, has been addressed by relaxing the assumption of controllability of the given dynamics, thus generalizing to the discrete time context the recent results proposed in Menini et al. (2012). Future work will concern the characterization of the conditions for the linear (feedback) equivalence of multi-input systems, the analysis of sampled data systems, and the study of the use of dynamic feedback laws.

\section{ACKNOWLEDGEMENTS}

The author whishes to thank Salvatore Monaco for the interesting discussions on the topic.

\section{REFERENCES}

E. Aranda-Bricaire, U. Kotta, C.H. Moog, Linearization of discrete-time systems SIAM J. Control Optim, 34 (6) pp.1999-2015, 1996.

A. Arapostathis, B. Jakubczyk, H-G. Lee, S.I.Marcus, E.D. Sontag, The effect of sampling on linear equivalence and feedback linearization, System and Control Letters, 13, pp.373-381, 1989.

Brockett, R.W. Feedback invariants for nonlinear systems Proc. IFAC Congress, Helsinki, pp. 1115-1120, 1978.

C. Califano, S. Monaco and D. Normand-Cyrot, On the problem of feedback linearization System and Control Letters, 36, pp.61-67, 1999.

C. Califano, L.A. Marquez-Martinez, C. Moog, On linear equivalence for time-delay systems, Proc. of the 2010 American Control Conference, ACC 2010, Baltimore, (MD), USA, Article N. 5531404, pp.6567-6572, 2010.
C. Califano, C. Moog, Feedback linear equivalence for nonlinear time delay systems, Proc. of the 50th IEEE Conference on Decision and Control and European Control Conference, CDC-ECC 2011; Orlando, FL, USA, Article N. 6161082, pp. 4158-4163, 2011

G. Cicogna, G. Gaeta, Symmetry and Perturbation Theory in Nonlinear Dynamics, Lecture notes in physics monographs, V.57, Springer, 1999.

J. W. Grizzle, A linear algebraic framework for the analysis of discrete-time nonlinear systems, SIAM J. Control Optim. 31, pp.1026-1044, 1993.

L.R. Hunt, R.Su, G. Meyer, Global transformation of nonlinear systems IEEE Transactions on Automatic Control, AC-28 (1), pp. 24-31, 1983.

A. Isidori, Nonlinear Control Systems, Springer, Berlin, 3rd ed, 1995.

B. Jakubczyk, Feedback linearization of discrete-time systems, Systems Control Lett. 9, pp.441-446, 1987.

B. Jakubczyk, W. Respondek, On linearization of control systems, Bull. Acad. Polonaise Sci. 28, pp.517-522, 1980.

A.J. Krener, On the equivalence of control systems and the linearization of nonlinear systems, SIAM J. of Contr., 11, pp.670-, 1973

A.J. Krener, A. Isidori, W. Respondek, Partial and robust linearization by feedback, Proc. IEEE CDC, pp. 126$130,1983$.

H.G. Lee, A. Arapostathis, S.I. Marcus, On the linearization of discrete-time systems, Int. J. Control, 45, pp.1783-1785, 1987.

R. Marino, On the largest feedback linearizable subsystem, Systems Control Lett., 6, pp.345-351, 1986.

L.Menini and A. Tornambè, Symmetries and semiinvariants in the analysis of nonlinear systems, Springer, London, 2011.

L.Menini and A. Tornambè, Exact and approximate feedback linearization without the linear controllability assumption, Automatica, 48, pp. 2221-2228, 2012.

S. Monaco, D. Normand-Cyrot, A unifying representation for nonlinear discrete-time and sampled dynamics, $J$. Math. Sys. Est. Contr. (Sum. 1995) 4 (7), 477-503, 1997.

S. Monaco, D. Normand-Cyrot, Minimum phase nonlinear discrete-time systems and feedback stabilization, Proc. 26th IEEE CDC, pp. 979-986, 1987.

S. Monaco, D. Normand-Cyrot, and C. Califano, Exponential representations of multi-input nonlinear discretetime dynamics, Proc. ACC-04, Boston, MA, pp. 49985003, 2004.

S. Monaco, D. Normand-Cyrot and C. Califano, From Chronological Calculus to Exponential Representations of Continuous and Discrete-Time Dynamics: A LieAlgebraic Approach, IEEE TAC, V.52 (12), pp. 22272241, 2007.

W. Respondek, Linearization, Feedback and Lie brackets, Prace Naukowe Instytutu Cybernetyki Technicznej, Politechniki Wroclawskiej (70), pp. 131-166, 1985

W. Respondek and I.A. Tall, On linearizability of strict feedforward systems Proceedings of the American Control Conference, art. no. 4586774, pp. 1929-1934, 2008

W. Respondek and I.A. Tall, Feedback linearizability of strict feedforward systems, Proceedings of the IEEE Conference on Decision and Control, art. no. 4739418, pp. 2499-2504, 2008 